\title{
AGREEMENT OF NHP AND SF-36 SOCIAL DOMAINS: AN EXPLORATORY STUDY
}

Bianca Macêdo de Alencar', Israel Souza ${ }^{2}$, Katia Nunes Sá ${ }^{3}$

Corresponding author: Bianca Macêdo de Alencar - biancamacedodealencar@gmail.com 'MSc in Tecnologies in Health. PhD candidate at BAHIANA - School of Medicine and Public Heath. Salvador, Bahia, Brazil. ${ }^{2} \mathrm{PhD}$ in Epidemiology in Public Health. Professor at the Federal Institute of Education, Science and Technology of Rio de Janeiro. Rio de Janeiro, Rio de Janeiro, Brasil. ${ }^{3} \mathrm{PhD}$ in Medicine and Human Health. Professor at BAHIANA - School of Medicine and Public Heath. Salvador, Bahia, Brazil.

\begin{abstract}
Introduction: Health Related Quality of Life (HRQoL) is an important outcome measure to investigate and monitor patients with chronic diseases. In order to achieve such goal, it is essential to choose an appropriate tool to evaluate the peculiarities of each population. Instruments to investigate HRQoL, NHP and SF-36 are alike in several aspects and are considered by the scientific community as interchangeable amongst each other. However, there are doubts regarding the agreement of their social domains. Objective: The present study aim to assess whether the social domains of the NHP and SF-36 correspond when applied in a population with chronic disease. Methods: The present research was made by applying the two instruments to a population with chronic disease. The social domains agreement was evaluated by Pearson correlation, ROC curve, AUC, Youdex Index, and Bland-Altman plot. Results: It was achieved a weak correlation between the two social domains $(r=0.3)$, confirmed by ROC curve with small AUC (0.416) and Youdex Index around 0.0. The Bland-Altman plot reaffirmed there is no agreement between the two subscale by achieving mean difference equal to 29.37 ( \pm 38.51 ), which made the concordance Interval vary from 106.39 to -47.65 . Conclusion: it was concluded that social domains of NHP and SF36 do not agree to each other. However, reasons for that are not explained and need to be investigated in futures studies. The current research was approved by the Research Ethics Committee of the BAHIANA - School of Medicine and Public Health under the protocol CAAE 516.42315 .5 .0000 .5544 .
\end{abstract}

Key-words: Data Accuracy; Quality of Life; Surveys and Questionnaires; 
Quality of life (QoL) is defined by WHO as "the individual's perception of their position in life in the context of culture and value systems in which they live, and in relation to their goals, expectations, standards and concerns"'. From this definition it is possible to notice that $Q$ oL concept is closely related to the context of culture and value system in which the individual lives. This understanding comes from the context in which the concept of QoL was first formulated: sociology and anthropology ${ }^{2}$. Originally, QoL was not studied by health sciences, but when such appropriation happened some elements were added to the inicial concept: functional losses, changes in relationships with society as a result of morbidity situation or physical limitation, the perception of the relationship with the health care system in its economic and political organization, as well as the value attributed by individuals to life expectancy when it is modified by the perception of physical or psychological constraints ${ }^{3}$.

Thus, to investigate Health Related Quality of Life (HRQoL), it is important to ascertain whether and to what extent morbidity is interfering with the individual's perception of their position in life, society and culture in which he/she lives, as well as his/hers objectives, expectations and concerns ${ }^{1}$. It is understandable therefore the great importance that the assessment of the social dimension have when investigating $H R Q \circ L$.

Among the instruments to assess general population HRQoL, the Nottingham Health Profile (NHP)4,5 and the Medical Outcomes Study 36-Item Short-Form Health Survey (SF-36) $)^{6,7}$ are well accepted by the scientific community and patients. Such instruments are alike in several aspects ${ }^{8,9}$ and are considered by the scientific community as replaceable each other. However, there were doubts regarding the agreement of their social domains $s^{6,8,10,11}$.

In order to clarify this question, there was made a systematic review that showed clear results concerning the correlation coefficients between social domains ${ }^{12}$. However, there are severe criticisms to this statistical technique when applied to compare two instruments. It is claimed that the correlation test is appropriate to assess the association between two variables.
However, its application to evaluate agreement among tools could hide biases and disagreements between them ${ }^{13}$. Accordingly, it should be used more appropriate statistical techniques to assess the agreement between assessment tools. However, it has not been explored by the authors reviewed.

Taking into consideration that the instruments in question (NHP and SF-36) have implications for HRQoL evaluation, it is clear that health professionals' need to have full understanding of them as evaluation tools, which makes it imperative to clarify the presented question. Aiming to fill this scientific gap, it was developed the current research: an exploratory study to assess whether the social domains of NHP and SF-36 correspond when applied in a population with chronic disease.

\section{METHODS}

It was conducted a study to test the agreement of SF36 and NHP social domains. The population was of individuals over 18 years with confirmed diagnostic of rheumatoid arthritis (RA), from a reference outpatient clinic for treatment of collagenosis, in the city of Salvador, Bahia, Brazil. Participants were interviewed between October 2011 and July 2012. The exclusion criteria covered limited understanding of the research instruments, or chronic degenerative comorbidities with potential to be confounders such as neurological, orthopedic, cardiac or pulmonary disorders.

Potentially includible individuals were invited to participate in the survey through telephone contact, when it was booked the first personal contact. This was followed by the presentation of research objectives with subsequent signing of the Informed Consent by those who agreed to participate. Data collection was made by primary source.

Participants visited three stations to respond the questionnaires through face to face interview in a private room. At each station there was an 
interviewer responsible for applying a questionnaire: the socio-demographic questionnaire, NHP and SF36. Each interviewer was responsible for applying the same questionnaire during the whole period of data collection in order to avoid collection bias. The interview was conducted by a previously trained staff to read the questionnaires how printed, without changing the words and without further explanation. For each questionnaire application a time of approximately 10 minutes was necessary, in accordance with the literature ${ }^{5,7}$.

The socio-demographic questionnaire consisted of a categorical variable about sex (male or female); age in years; color of skin categorized in white, red, black or brown; socio-economic class categorized as $A 1, A 2, B 1, B 2, C 1, C 2$ and $D$ according to $A B E P$ (Associação Brasileira de Instituto de Pesquisa de Mercado); schooling was classified as below and above eight years of study.

Using data from SF-36 and NHP, it was possible to get the domain scores of these scales, including social isolation (NHP) and social functioning (SF36). The NHP is a generic tool to assess HRQoL, originally developed for patients with some chronic involvement. In order to address the various aspects of HRQoL, the instrument investigates six different areas: Energy (EN/NHP), Pain (P/NHP), Emotional Reactions (ER/NHP), Sleep (SL/NHP), Social Isolation (SI/NHP) and Physical Mobility (PM/NHP). NHP is composed of 38 dichotomous questions, to which a point is scored for each affirmative answer. Thus 38 points can be scored, which indicates a worse HRQoL condition compared to the individual who scores zero. The same way of calculating can be applied to the domains, which helps identify areas in which the individual is less or more committed.5 For comparison with SF-36, scores can be transformed into a score ranging from 0 to 100 , which is often adopted.

The SF-36 is equally a generic instrument to evaluate HRQoL. It consists of 36 questions with different response options; some are dichotomist; while others are Likert scale model, up to six options of intensity. The instrument investigates eight domains related to HRQoL: Physical Functioning (PF/SF-36) Role Physical Limitation (PL/SF-36), Pain (P/SF-36) General Health Perception (GH/SF-36) Energy/Vitality (VT/SF-36), Social Functioning (SF/SF-36), Role
Emotional Limitation (EL/SF-36) and Mental Health (MH/SF-36). A mathematical formula proposed by SF-36 creators allows computing participants' scores. The total score relates to the individual's HRQoL. However, the calculation can be performed individually per domain. Score ranges from 0 (worst score) to 100 (best score) ${ }^{7}$.

Both questionnaires should preferably be selfadministered $^{5,7}$. The application by interview is susceptible to collecting bias, especially when the questions are too long or subjective. However faceto-face interview is acceptable in case of illiterate or functionally illiterate individuals, provided that all care is taken for the interviewer does not suggest the respondent answer. In this research, it was decided to apply the questionnaires to all patients through interviews to better standardization.

For the sample calculation 0.70 correlation between social subscales was used. This value is the minimum considered as a good correlation in the studies reviewed ${ }^{8,14-19}$. Thereby using $G *$ Power 3.1.9.2 software, alpha of $5 \%$, power of $80 \%$ and 0.70 correlation, it was calculated a sample of 84 subjects. From the service records, with 456 registered patients, 97 participants were randomly selected using a table of random numbers. If the individual contacted was not found or did not want to participate, the next table number was included in the list of participants until the sample reached the estimated size.

Data were analyzed using SPSS (17.0). The sociodemographic variables were treated in absolute numbers and percentages or average and standard deviation, since it has little transgressed the normal distribution of frequency. Adopting alpha $5 \%$ and $80 \%$ power for all analyzes, the Pearson correlation coefficient of SF-36 and NHP domains was calculated in order to investigate whether the social areas of NHP and SF-36 correlated. The Cronbach's alpha of the two scales social domains was also calculated to assess the internal consistency. It was made the ROC curve of Social Isolation (NHP) taking as reference line Social Functioning (SF-36); and it was calculated the Area Under the Curve (AUC) in order to verify the sensitivity and specificity of a domain relative to each other. Subsequently, the Youden index was calculated to estimate the sensitivity and specificity of Social Functioning (SF-36) domain cutoffs. 
The Youden index indicates the lower total proportion of possible misclassification, it is the cut-off point with the lowest number of incorrect diagnoses (false positives added to false negatives). The index ranges from - 1 to +1 . The calculation can be performed manually:

$$
\mathrm{J}=1-(\alpha+\beta)=1-[(1-(\text { sensitivity })+(1-(\text { specificity })]=(\text { sensitivity })+(\text { specificity })-1
$$

If the test does not have a diagnostic value, $\mathrm{J}=0$. If the test is invariably correct, $\mathrm{J}=1$. Values between - 1 and 0 indicates that the test is negatively associated with the diagnosis. Values closer to +1 indicates the better method ${ }^{20}$.

Afterwards, it was built Bland-Altman plot $^{13}$ to assess agreement between the two instruments. For this evaluation, the scores of SI/NHP were reversed, so that the social domains of the SF-36 and NHP scored zero to the participant with the worst possible performance in sub-scale and 100 to the individual with the best possible performance.

The graph is made from the representation of the extent of each individual. The vertical axis is equal to the difference of the measures offered by each instrument to the same individual, while the horizontal axis indicates the arithmetic mean of these measures. Crossing the information of the two axis, it marks the graph points. Then it is computed the mean and standard deviation values of the vertical axis. A horizontal line is made corresponding to the value of this average. Other 2 lines are made equivalent to mean values plus standard deviation 1.96, which corresponds to $95 \%$ confidence limits (limits of agreement - LOA), considering a normal distribution.

This research is part of the project entitled General Clinical Profile of Patients with Rheumatoid Arthritis from a Reference Clinic in Salvador, Bahia, approved by the Research Ethics Committee of Bahiana School of Medicine and Public Health under the protocol CAAE 516.42315.5.0000.5544.

The study followed the definitions of Resolution number 466/12 of the Brazilian National Council of Health for Research in Humans, guaranteeing anonymity, non-maleficence and beneficence to participants. The biggest benefit generated by the project is the best knowledge of this population profile for future interventions for their wellbeing. In addition, the current research allows better knowledge of the available tools to evaluate the social aspect of HRQoL. Furthemore, it was offered as a directly and immediately benefit to the volunteers, a lecture given about their disease, $R A$, as well as the daily care needed to maintain a good quality of life.

\section{RESULTS}

The sample consisted of all patients registered in a Reference Clinic for Treatment of Collagen, in the city of Salvador, Bahia, Brazil, who met the inclusion criteria. There were no refusals and only three patients were not found from the chart data. As a result, data were collected from 97 patients with confirmed diagnosis of RA. The sample was characterized by women $(92.8 \%)$, mean age of 52.5 years old $(\mathrm{SD} \pm 11)$, brow skin $(47.4 \%), \mathrm{C} 2$ socio-economic class $(39.2 \%)$, having studied for eight years or more $(64.9 \%)$ (Table 1$)$. There were no missing data.

Table 1. Sociodemographic characteristics of individuals with Rheumatoid Arthritis, accompanied in a reference unit for the treatment of collagen in the city of Salvador, Bahia, 2011-2012 ( $\mathrm{N}=97)$.

\begin{tabular}{lcc}
\hline Variables & $\mathbf{n}$ & $\%$ \\
& Average & or SD \\
\hline Gender & & \\
Female & 90 & 92.8 \\
& & \\
Age & 52.5 & 11.0 \\
Skin Color & & \\
White & & \\
Red & 11 & 11.3 \\
Black & 03 & 03.1 \\
Brow & 37 & 38.1 \\
Social Class & 46 & 47.4 \\
A1 & & \\
A2 & 03 & 03.1 \\
B1 & 02 & 02.1 \\
B2 & 03 & 03.1 \\
C1 & 17 & 17.5 \\
C2 & 24 & 24.7 \\
D & 38 & 39.2 \\
Schooling & 10 & 10.3 \\
Less than 8 years of study & & \\
8 years of study or more & 34 & 35.1 \\
\hline Source: Research Protocol & 63 & 64.9 \\
\hline
\end{tabular}


Regarding the psychometric evaluation of tested tools, the assessment of internal consistency by Cronbach's alpha, SF/SF-36 domain showed $\alpha=0.50$, while SI/ NHP hit $\alpha=0.73$ (Table 2). When calculating the Pearson correlation to verify the convergent validity between social isolation dimension of NHP and social functioning of SF-36, it revealed a significant positive correlation ( $r=0.305 ; p=0.006)$, opposite of expected. Furthermore It was found a higher correlation between SI/NHP and MH/SF-36 ( $r=$ $0.433, p<0.001$ ), this time a negative value, as expected. The results of correlations between all areas of NHP and SF-36, especially the theoretically expected correlations, are presented in Table 3.
Table 2. Internal Consistency of SF-36 and NHP comparable domains when applied to a population with Rheumatoid Arthritis, accompanied in a reference unit for the treatment of collagen in the city of Salvador, Bahia, 2011-2012.

\begin{tabular}{cc}
\hline SF-36 Domains & Cronbach's Alfa \\
\hline Pain & 0.649 \\
Physical Functioning & 0.812 \\
Role Emotional Limitation & 0.753 \\
Energy/Vitality & 0.651 \\
Social Functioning & 0.506 \\
\hline NHP Domains & \\
\hline Pain & 0.759 \\
Physical Mobility & 0.548 \\
Emotional Reactions & 0.847 \\
Energy & 0.647 \\
Social Isolation & 0.730
\end{tabular}

Source: Research Protocol

Table 3. NHP and the SF-36 correlation when applied to a population with Rheumatoid Arthritis, accompanied in a reference unit for the treatment of collagen in the city of Salvador, Bahia, 201 1-2012.

\begin{tabular}{|c|c|c|c|c|c|c|c|c|c|c|c|c|}
\hline & \multicolumn{2}{|c|}{ Energy } & \multicolumn{2}{|c|}{ Peain } & \multicolumn{2}{|c|}{$\begin{array}{l}\text { Emotional } \\
\text { Reactions }\end{array}$} & \multicolumn{2}{|c|}{ Sleep } & \multicolumn{2}{|c|}{ Social Isolation } & \multicolumn{2}{|c|}{$\begin{array}{l}\text { Physical } \\
\text { Mobility }\end{array}$} \\
\hline & $\mathbf{r}$ & $\mathbf{p}$ & $\mathbf{r}$ & $\mathbf{p}$ & $\mathbf{r}$ & $\mathbf{p}$ & $\mathbf{r}$ & $\mathbf{p}$ & $\mathbf{r}$ & $\mathbf{p}$ & $\mathbf{r}$ & $\mathbf{p}$ \\
\hline Physical Functioning & $-.583^{\circ}$ & .000 & $-.596^{\circ 0}$ & .000 & $-.315^{\circ 0}$ & .005 & -.205 & .070 & -.196 & .084 & $-.638^{\infty}$ & .000 \\
\hline Role Physical Limitation & $-.407^{\circ+}$ & .000 & $-.318^{+\infty}$ & .004 & $-.247^{\circ}$ & .028 & -.071 & .534 & -.194 & .086 & $-.240^{\circ}$ & .033 \\
\hline Pain & .077 & .498 & .210 & .063 & .156 & 17 & .027 & .814 & .073 & .522 & -.059 & .604 \\
\hline $\begin{array}{l}\text { General Health } \\
\text { Perception }\end{array}$ & -.065 & .572 & -.092 & .422 & -.143 & .208 & .221 & .051 & .051 & .655 & -.058 & .614 \\
\hline Vitality & $-.362^{\infty}$ & .001 & $-.298^{\circ+}$ & .008 & $-.391^{\circ 0}$ & .000 & $-.281^{\circ}$ & .012 & -.163 & .150 & $-.243^{\circ}$ & .031 \\
\hline Social Functioning & .031 & .789 & -.194 & .087 & .123 & .281 & -.153 & .178 & $.305^{\infty}$ & .006 & .020 & .859 \\
\hline Role Emotional Limitation & -.163 & .151 & -.047 & .681 & $-.316^{\circ}$ & .005 & -.030 & .792 & $-.369^{\circ \circ}$ & .001 & -.167 & .140 \\
\hline Mental Health & $-.305^{\circ *}$ & .006 & $-.329^{\circ+}$ & .003 & $-.635^{\infty}$ & .000 & $-.232^{\circ}$ & .040 & $-.433^{\circ 0}$ & .000 & $-.229^{\circ}$ & .042 \\
\hline
\end{tabular}

The ROC curve of Social Isolation (NHP) compared to Social Functioning domain (SF-36) showed AUC of $0.416(\mathrm{SE}=0.8)(p$-value $=0.28)$ (Figure 1$)$, indicating low sensitivity and specificity of a domain to each other. When checking the Youden index, It was noted that most of the scores showed low sensitivity and low specificity rating close to zero or negative, indicating that the tolls do not discriminate well each other (table 4).

Table 4. Sensitivity and specificity of cutoff points of Social Isolation (NHP) in relation to Social Functioning (SF-36) when applied to a population with Rheumatoid Arthritis, accompanied in a reference unit for the treatment of collagen in the city of Salvador, Bahia, 2011-2012.

\begin{tabular}{|c|c|c|c|c|}
\hline Variable & Escors & Sensitivity & 1 - Specificity & Youden index \\
\hline & -1.0000 & 1.000 & 1.000 & 0.000 \\
\hline & 10.0000 & .944 & .967 & -0.023 \\
\hline & 30.0000 & .889 & .934 & -0.046 \\
\hline & 50.0000 & .611 & .836 & -0.225 \\
\hline & 70.0000 & .556 & .738 & -0.182 \\
\hline & 90.0000 & .444 & .325 & -0.080 \\
\hline Social Isolation & 101.0000 & 0.000 & 0.000 & 0.000 \\
\hline
\end{tabular}

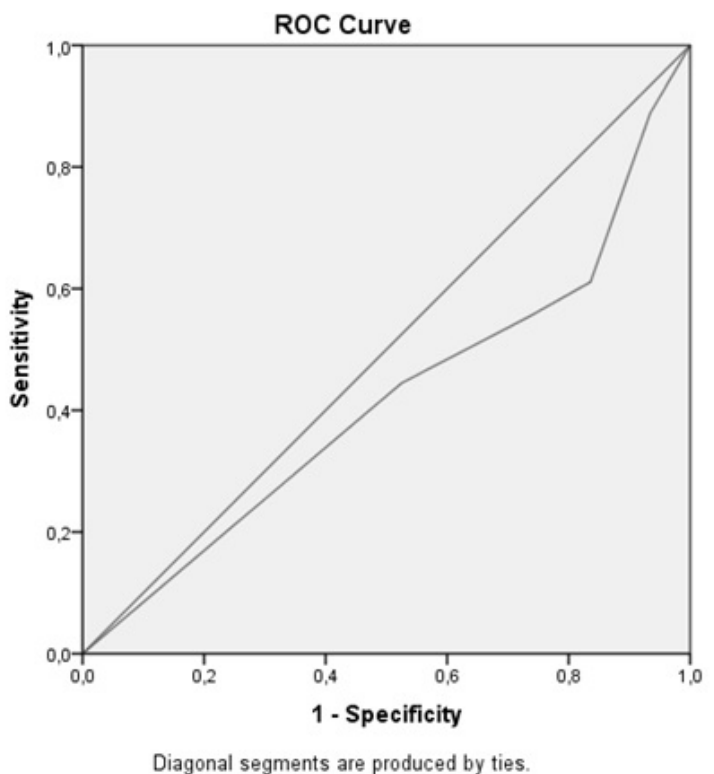

Source: Research Protocol
Area Under the Curve $=0.416$
Standard Error: 0.082
p value: 0.282
Confidence Interval: $0.256-0.577$

Figure 1. ROC curve of Social Isolation (NHP) in reference to Social Functioning (SF-36) when applied to a population with Rheumatoid Arthritis, accompanied in a reference unit for the treatment of collagen in the city of Salvador, Bahia, 2011-2012. 
Bland-Altman plot, comparing NHP and SF-36 social domains, showed mean difference equal to 29.37 $( \pm 38.51)$, and LOA from -47.65 to 106.39. Three outliers were found (Figure 2).

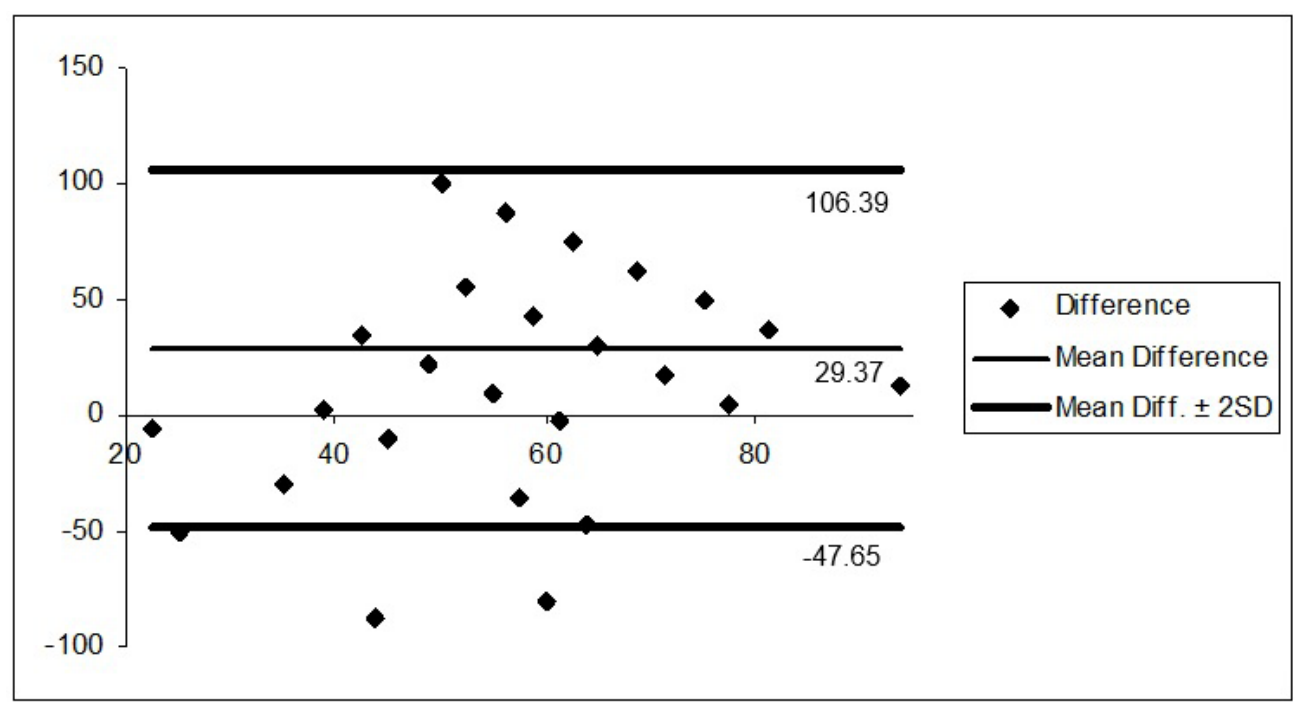

Vertical axis: difference between SF-36 and NHP measures

Average of difference $=29.37$

Standard Deviation $=38.51$

Concordance Interval $=106.39$ to -47.65

Horizontal axis: average of SF-36 and NHP measures

Figure 2. Bland-Altman plot for evaluation of Social Functioning (SF-36) and Social Isolation (NHP) agreement, when applied to a population with Rheumatoid Arthritis accompanied in a reference unit for the treatment of collagen in the city of Salvador, Bahia, 2011-2012.

\section{DISCUSSION}

The NHP and SF-36 instruments are designed to evaluate the HRQoL and have been treated in the scientific literature as comparable, replaceable one another. However, the current study demonstrated that NHP and SF-36 social domains do not match when applied to a population with RA accompanied in a reference unit for the treatment of collagen in the city of Salvador, Bahia, between the months October 2011 to July 2012.

The first psychometric property tested to answer the research question, was internal consistency. It was necessary to know whether the instruments assessed what each of them intended to, before checking if they evaluated the same thing. In this exploratory study SI/NHP had good internal consistency, which corroborates previously published studies $8,10,14,16$.

However, SF/SF-36 showed moderate internal consistency, which was also observed by some authors who investigated hemodialysis patients 8 or ischemia of the lower limbs ${ }^{16}$. Though, SF/SF-36 showed good internal consistency when assessed by other researchers who have investigated patients with sensory hyperreactivity ${ }^{14}$, patients after stroke ${ }^{21}$, physically active elderly ${ }^{10}$, patients with hereditary neuromuscular disease ${ }^{11}$, among others ${ }^{6,17}$, Therefore, considering the results of current study and previously published evidence, both domains may be considered to have moderate to good internal consistency. This may be mutable according to limitations imposed by different kinds of disease.

Regarding convergent validity, the first test used was the correlation coefficient, employed in previous researches that sought to evaluate this property ${ }^{6,8-11,14-19,21-25}$. The literature registered repeatedly, low convergence between social domains of SF-36 and NHP8,14-19,21,24,25. However, in RA population, presently studied, the Pearson correlation coefficient showed a result that had not been observed in previous studies, the positive correlation, opposite to what was expected since the 
scales are inverse to each other. This result indicated that, in addition to the two domains little agreement in their assessments, they can still be divergent. It is, as an instrument evaluates individuals with RA to have good quality of life in relation to its social aspect, the other instrument can make a contrary assessment of the same individual, indicating a poor quality of life.

This research also adopted other ways to evaluate the agreement of the two subscales concerned. The first was the ROC curve, where the representative line of sensitivity and specificity of SI/NHP relative to SF/SF-36 domain was below the reference line; consequently, culminating in a low AUC. This graphical display shows low sensitivity and specificity of SI/NHP relative to SF/SF-36. In other words, low convergence between the two subscales. Similarly to the direct correlation that was expected to be the opposite, inversely correlated. This shows that while a sub-scale indicates good quality of life for a person, the other evaluates the same subject as having bad quality of life in relation to the social domain. The low agreement between social areas studied is reaffirmed by the Youden index, in being equal or close to zero, it indicates that $\mathrm{SI} / \mathrm{NHP}$ is not related to the evaluation of SF/SF-36.

In the evaluation performed from Bland-Altman plot, there was a broad bias on the two instruments agreement. With average of differences 29.37, instruments were highly different from each other. In Bland-Altman evaluation it is expected average of differences close to zero; it is, the smallest possible divergence. The average value of differences indicates the value of the constant bias, it is 29.37 in this measure. The high value of the standard deviation (38.51), even greater than the average value, exacerbates the divergence. This, therefore, led the upper limit of the correlation interval reaches the value of 106.39. Such high concordance limit is unacceptable, since the scales scores from 0 to 100 . That is, even if a scale rates the individual with its minimum value $(0)$ and the other with its maximum value (100) the difference 106.39 would not be achieved. This graphic layout indicates that there was no agreement between the methods, but there is systematic error between the evaluations of $\mathrm{SI} /$ NHP and SF/SF-36.

The results currently presented are clear, there is no statistical convergence between the social domains of NHP and SF-36 when applied to a population with RA from a referral center for treatment of collagen in Salvador, Bahia in the period of October 2011 to July 2012 . Assumed comparability of these domains is inconsistent. The question is: why? For what reason social domains of HRQoL instruments would generate different evaluations? It is speculated that the content of the two subscales are different ${ }^{11}, \mathrm{SI} / \mathrm{NHP}$ may investigate psychological aspects 16 related to the presence of depression8 or the difficulty of contacting because of cognitive-behavioral changes ${ }^{17}$, while SF/SF-36 may investigate the social question itself $f^{8,16,17}$. The highest correlation between SI/NHP and MH/SF-36 may shows that any of these hypothesis, or all of them in a complementary manner may be true. However, there were no publications about the investigation of any of these hypothesis, leaving such a scientific gap.

The methodology used in this exploratory study had the main advantage of allowing the statistical strategies variety used. Tests presented results that corroborated each other and corroborated with other studies, suggesting no agreement between SF36 and NHP social domains. However, it is limited by not allowing understand this divergence cause.

\section{CONCLUSION}

The investigation of the assumed comparability of NHP and SF-36 social domains, by a quantitative method of analysis of questionnaires applied to a population with RA from a reference unit for the treatment of collagen in the city of Salvador, Bahia, from October 2011 to July 2012 and subsequent use of the Pearson correlation coefficient tests, Youden index, ROC curve, AUC and Bland-Altman plot, indicated no agreement of the two investigated domains.

\section{ACKNOWLEDGEMENTS}

This research was funded by Programa de Suporte à Pós-Graduação de Instituições de Ensino Particulares (PROSUP) of Coordenação de Aperfeiçoamento de Pessoal de Nível Superior (CAPES) in the form of doctoral scholarship to the main author. 


\section{AUTHOR CONTRIBUTIONS}

Alencar BM was responsible for the investigation question formulation, data interpretation, background research and text writing. Souza I was responsible for the statistical analises and interpretation. Sá KN was responsible for the original study on which the present article is based, data interpretation and text revision.

\section{COMPETING INTERESTS}

No financial, legal or political competing interests with third parties (government, commercial, private foundation, etc.) were disclosed for any aspect of the submitted work (including but not limited to grants, data monitoring board, study design, manuscript preparation, statistical analysis, etc.).

\section{REFERENCES}

1. Saxena S, Orley J, Group W. Quality of life assessment : the World Health Organization perspective. Eur Psychiatry. 1997;1 2(Suppl 3):263-6. doi: 10.1016/S09249338(97)89095-5

2. Minayo MC de S, Hartz ZM de A, Buss PM. Qualidade de vida e saúde: um debate necessário. Cien Saude Colet. $2000 ; 5(1): 7-18$. doi: 10.1590/S1413$\underline{81232000000100002}$

3. Owolabi MO. Health-related quality of life (HRQOL) measures: there are still many unanswered questions about human life. ScientificWorldJournal. 2008; 14:357-63. doi: $10.1100 /$ tsw.2008.54

4. Hunt SM, McKenna SP, McEwen J, Backett EM, Williams J, Papp E. A quantitative approach to perceived health status: a validation study. J Epidemiol Community Heal. 19801;34(4):281-6. doi: 10.1136/iech.34.4.281

5. Teixeira-Salmela LF, Magalhães $L$ de $C$, Souza AC, Lima $M$ de C, Lima RCM, Goulart F. Adaptação do Perfil de Saúde de Nottingham: um instrumento simples de avaliação da qualidade de vida. Cad Saude Publica. 2004;20(4):905-14. doi: $10.1590 /$ S0102-311X2004000400004

6. Brazier JE, Harper R, Jones NM, O'Cathain A, Thomas $\mathrm{KJ}$, Usherwood T et al. Validating the SF-36 health survey questionnaire: new outcome measure for primary care. BMJ. 1992;305(6846):160-4. doi: doi.org/10.1136/ bmi.305.6846.160

7. Ciconelli RM, Ferraz MB, Santos W, Meinão I, Quaresma $M R$. Tradução para a língua portuguesa e validação do questionário genérico de avaliação de qualidade de vida SF-36 (Brasil SF-36). Rev Bras Reumatol. 1999;39(3):143-50.

8. Zengin N, Ören B, Gül A, Üstündag H. Assessment of quality of life in haemodialysis patients: a comparison of the
Nottingham Health Profile and the Short Form 36. Int J Nurs Pract. 2014;20(2):115-25. doi: $10.1111 /$ ijn.12130

9. Cabral DL, Laurentino GEC, Damascena CG, Faria CDCM, Melo PG, Teixeira-Salmela LF. Comparisons of the Nottingham Health Profile and the SF-36 health survey for the assessment of quality of life in individuals with chronic stroke. Brazilian J Phys Ther. 2012;16(4):301-8. doi: 10.1590/S141335552012005000029

10. Jagsch $R$, Pils K. Which instrument is more suitable to assess health-related quality of life: Nottingham Health Profile or Short-Form-36? Wien Med Wochenschr. 2006;24];156(5-6):149-57. doi: 10.1007/s10354-0060266-9

11. Boyer F, Morrone I, Laffont I, Dizien O, Etienne JC, Novella JL. Health related quality of life in people with hereditary neuromuscular diseases: an investigation of testretest agreement with comparison between two generic questionnaires, the Nottingham health profile and the short form-36 items. Neuromuscul Disord. 2006; 16(2):99-106. doi: $10.1016 /$ i.nmd.2005.11.002

12. Alencar BM, Araújo PCS, Sá KN. Concordância entre os domínios sociais do PSN E SF-36 na avaliação da qualidade de vida: uma revisão sistemática da literatura. Rev Diálogos Possíveis. 2015;14(1):1-26.

13. Hanneman SK. Design, Analysis and Interpretation of Method-Comparison Studies. AACN Adv Crit Care. 2008;19(2):223-34. doi: 10.1097/01. AACN.0000318125.41512.a3

14. Kristofferzon M-L, Ternesten-Hasséus E. A study of two generic health-related quality of life questionnaires-Nottingham Health Profile and Short-Form 36 Health Survey-and of coping in patients with sensory hyperreactivity. Health Qual Life Outcomes. 2013;11:182. doi: 10.1186/1477$\underline{\text { 7525-11-182 }}$

15. Hagel S, Lindqvist E, Petersson I, Nilsson J-A, Bremander A. Validation of outcome measurement instruments used in a multidisciplinary rehabilitation intervention for patients with chronic inflammatory arthritis: Linking of the International Classification of Functioning, Disability and Health, construct validity a. J Rehabil Med. 2011 ;43(5):41 1-9. doi: 10.2340/16501977-0794

16. Wann-Hansson C, Hallberg IR, Risberg B, Klevsgård R. A comparison of the Nottingham Health Profile and Short Form 36 Health Survey in patients with chronic lower limb ischaemia in a longitudinal perspective. Health Qual Life Outcomes. 2004;2:9. doi: 10.1 186/1477-7525-2-9

17. Meyer-Rosberg K, Burckhardt CS, Huizar K, Kvarnström a, Nordfors LO, Kristofferson a. A comparison of the SF-36 and Nottingham Health Profile in patients with chronic neuropathic pain. Eur J Pain. 2001;5(4):391-403. doi: 10.1053/ evip.2001.0260 
18. Prieto L, Alonso J, Ferrer M, Antó JM. Are results of the SF-36 health survey and the nottingham health profile similar?: A comparison in COPD patients. J Clin Epidemiol [Internet]. 1997;50(4):463-73. doi: 10.1016/S08954356(96)00420-9

19. Stansfeld SA, Roberts R, Foot SP. Assessing the validity of the SF-36 General Health Survey. Qual Life Res. $1997 ; 6(3): 217-24$.

20. Jalali $R$, Rezaie M. Predicting pressure ulcer risk: comparing the predictive validity of 4 scales. Adv Skin Wound Care. 2005;18(2):92-7.

21. Unalan D, Soyuer F, Ozturk A. Should the Nottingham Health Profile or the Short Form-36 be given preference in stroke? Neurociences. 2009; 14(1):45-52.

22. Faria CDCM, Teixeira-Salmela LF, Nascimento VB, Costa AP, Brito ND, Rodrigues-De-Paula F. Comparação dos instrumentos de qualidade de vida Perfil de Saúde de Nottingham e Short Form-36 em idosos da comunidade for assessing the quality of life of community-dwelling elderly. $2011 ; 15(5): 399-405$.

23. Ozalevli S, Karaali H, Cankurtaran F, Kilinc O, Akkoclu A. Comparison of Short Form-36 Health Survey and Nottingham Health Profile in moderate to severe patients with COPD. J Eval Clin Pract. 2008;14(4):493-9. doi: 10.1111/i.13652753.2007.00904.x

24. McQueen DA, Long MJ, Schurman JR. Selecting a subjective health status measure for optimum utility in everyday orthopaedic practice. J Eval Clin Pract. $2005 ; 11(1): 45-51$.

25. Crockett AJ, Cranston JM, Moss JR, Alpers JH. The MOS SF-36 health survey questionnaire in severe chronic airflow limitation: Comparison with the Nottingham health profile. Qual Life Res. 1996;5(3):330-8. 